\title{
Association between Medical Costs and the ProVent Model in Patients Requiring Prolonged Mechanical Ventilation
}

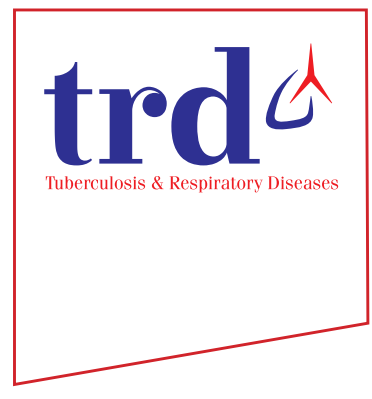

\author{
Jiyeon Roh, M.D. ${ }^{1}{ }^{(\mathbb{D}}$, Myung-Jun Shin, M.D. ${ }^{2}{ }^{\circledR}$, Eun Suk Jeong, R.N. ${ }^{1}$ and Kwangha Lee, M.D., Ph.D. ${ }^{1}$ (iD \\ ${ }^{1}$ Division of Pulmonary, Allergy, and Critical Care Medicine, Department of Internal Medicine, ${ }^{2}$ Department of Rehabilitation \\ Medicine, Pusan National University School of Medicine, Busan, Korea
}

Background: The purpose of this study was to determine whether components of the ProVent model can predict the high medical costs in Korean patients requiring at least 21 days of mechanical ventilation (prolonged mechanical ventilation $[\mathrm{PMV}])$.

Methods: Retrospective data from 302 patients (61.6\% male; median age, 63.0 years) who had received PMV in the past 5 years were analyzed. To determine the relationship between medical cost per patient and components of the ProVent model, we collected the following data on day 21 of mechanical ventilation (MV): age, blood platelet count, requirement for hemodialysis, and requirement for vasopressors.

Results: The mortality rate in the intensive care unit (ICU) was $31.5 \%$. The average medical costs per patient during ICU and total hospital (ICU and general ward) stay were 35,105 and 41,110 US dollars (USD), respectively. The following components of the ProVent model were associated with higher medical costs during ICU stay: age <50 years (average 42,731 USD vs. 33,710 USD, $\mathrm{p}=0.001)$, thrombocytopenia on day 21 of MV (36,237 USD vs. 34,783 USD, $\mathrm{p}=0.009)$, and requirement for hemodialysis on day 21 of MV (57,864 USD vs. 33,509 USD, $\mathrm{p}<0.001)$. As the number of these three components increased, a positive correlation was found betweeen medical costs and ICU stay based on the Pearson's correlation coefficient $(\gamma)(\gamma=0.367, \mathrm{p}<0.001)$.

Conclusion: The ProVent model can be used to predict high medical costs in PMV patients during ICU stay. The highest medical costs were for patients who required hemodialysis on day 21 of MV.

Keywords: Costs; Mechanical Ventilation; Intensive Care Units

Address for correspondence: Kwangha Lee, M.D., Ph.D.

Division of Pulmonary, Allergy, and Critical Care Medicine, Department of Internal Medicine, Pusan National University School of Medicine, 179 Gudeok-ro, Seo-gu, Busan 49241, Korea

Phone: 82-51-240-7743, Fax: 82-52-245-3127

E-mail: jubilate@pusan.ac.kr

Received: Aug. 7, 2018

Revised: Oct. 10, 2018

Accepted: Oct. 29, 2018

Published online: Dec. 20, 2018

(c) It is identical to the Creative Commons Attribution Non-Commercial License (http://creativecommons.org/licenses/by-nc/4.0/). The Korean Academy of Tuberculosis and Respiratory Diseases.

\section{Introduction}

Advances in critical care intervention have led to improvements in short-term survival for intensive care unit (ICU) patients with acute critical illness ${ }^{1}$. However, a proportion of patients who survive the first few days of acute catastrophic illness become dependent on prolonged mechanical ventilation $(\mathrm{PMV})^{1}$. Currently, PMV is defined as a minimum of 21 days of invasive mechanical ventilation (MV) ${ }^{2}$. As a result of improvements in critical care medicine, the number of patients receiving PMV has increased in recent years ${ }^{1}$. These patients display neuromuscular weakness, recurrent infections with multidrug-resistant bacteria, and delirium ${ }^{1,3}$. As a result, they require various medical resources for a long period of time in an ICU, which is associated with high medical costs. Although the economic burden of PMV patients in Western countries has been investigated ${ }^{1,4,5}$, data on the medical costs of PMV 
patients in Korea are sparse.

To identify PMV patients with a high 1-year mortality risk, the Prognosis for Prolonged Ventilation (ProVent) model ${ }^{6,7}$, which estimates the 1-year mortality rate of patients receiving at least 21 days of MV, was developed. The performance of the ProVent score has since been validated in several patient populations $^{8,9}$. In addition to predicting mortality, specific components of the model, such as the requirement for hemodialysis or vasopressors, are associated with high consumption of medical resources. Therefore, a plausible hypothesis is that the ProVent model could be used to predict high medical costs during ICU and total hospital stay in patients requiring PMV. Such prediction would facilitate ICU management decisions and communication between attending physicians and the patient and/or their relatives.

We investigated whether components of the ProVent model are predictive of high medical costs during ICU and total hospital stay in Korean patients requiring PMV.

\section{Materials and Methods}

\section{Study design and patient selection}

This retrospective study was performed in the ICUs of a 1,100-bed university-affiliated tertiary care hospital in Busan, Korea. This hospital has six functionally separate ICUs with a total of 85 beds (medical, 12 beds; surgical, 10 beds; cardiostroke, 14 beds; neurosurgical, 13 beds; emergency, 20 beds; trauma, 16 beds). All of the ICUs have full cardiovascular and close airway monitoring equipment, as well as one full-time ICU specialist; the nurse-to-bed ratio is 1:3. All patients are managed according to the lung-protective ventilator strategy ${ }^{10}$. In this study, the inclusion period was from January 1, 2011 to December 31,2015 . Survival status in all patients was obtained until December 31 2016. Adult patients who were admitted to the ICUs and received MV for at least 21 days were retrospectively enrolled in the analyses. Patients with chronic diseases requiring MV (either invasive or noninvasive) before ICU admission were not initially screened. The exclusion criteria were as follows: age $<18$ years, irreversible brain injury, acute or chronic neuromuscular disease, long-term MV before ICU admission, and lack of data on patient characteristics or outcomes. These inclusion or exclusion criteria were identical to those used in previous studies ${ }^{7-9}$. All investigators contributed to the design of the study and confirmed that the objectives and procedures were honestly disclosed. We could not determine the exact sample size because the study was retrospective. In May and October 2017, the electronic medical records of all enrolled patients were reviewed retrospectively. The relevant medical, laboratory, and radiological data were extracted and used to complete a case report form for each patient. The data in these reports were analyzed. Our study had no impact on the treatment of patients in the study.

\section{Data collection}

The following demographic and clinical data were gathered from the medical records of each patient: age, sex, ICU admission diagnosis, and comorbidities prior to ICU admission based on Charlson Comorbidity Index ${ }^{11}$. The severity of illness within 24 hours of ICU admission was measured using the Acute Physiology and Chronic Health Evaluation (APACHE) II score, and concomitant organ failure was measured according to the Sequential Organ Failure Assessment (SOFA) score ${ }^{12,13}$. We also assessed the following data: ICU and hospital length of stay (LOS), duration of MV, ICU and hospital cumulative mortality rates, and 1-year cumulative mortality rate after day 21 of MV. In addition, the main department of all patients was determined and categorized as medical (internal medicine, neurology, rehabilitation medicine, and emergency medicine) or surgical (trauma, general surgery, cardiothoracic surgery, neurosurgery, and obstetrics/gynecology). In addition, we evaluated the tracheostomy status prior to day 21 of MV. For patients discharged alive from hospital, the 1-year mortality rate after day 21 of MV was obtained from the National Health Insurance Service Database. To determine the association between medical cost per patient and components of the ProVent model $^{6,7}$, we collected the following data on day 21 of MV: age, blood platelet count, requirement for hemodialysis (defined as use of any form of renal replacement therapy on or within 48 hours of day 21 of MV), and requirement for vasopressors. For all enrolled patients, total medical costs for all medical resources used (including all medicines) during ICU and hospital (ICU and general ward) stay were retrieved with the permission of the institutional review board (IRB). The cost of each medical intervention was estimated in accordance with the reimbursement scales of the Korean Health Insurance Review and Assessment Service. All of the costs are presented in US Dollars (USD) at an exchange rate of 1 USD equaling 1,115.50 Korean won (exchange rate on November 4, 2017).

\section{Statistical analyses}

Continuous variables are expressed as median (range). Categorical variables are presented as number (\%). Depending on the normality of the respective distribution, the Student's t-test or Mann-Whitney U test was used for comparisons of continuous variables. One-way analyses of variance or the Kruskal-Wallis test was used for three-group comparisons of continuous variables. The chi-square or Fisher exact test (for small numbers) was used to compare categorical variables. To evaluate the relationship between total medical costs per person and components of the ProVent model, Pearson's correlation coefficient $(\gamma)$ was calculated. To evaluate factors 
independently predictive of ICU and hospital mortality, logistic regression analyses were performed on all components of the ProVent model. Model discrimination was assessed by the area under the receiver operating characteristic curve (AUC), and model calibration was assessed using the Hosmer-Lemeshow test. The $\beta$-coefficient values derived from multiple logistic regressions were simplified as natural numbers $>0$, and the ProVent models for predicting ICU and hospital mortality were calculated as the sum of these simplified $\beta$-coefficient values, which was same as the previous studies ${ }^{7,14}$. All tests were two-tailed, and values of $\mathrm{p}<0.05$ were considered statistically significant. All analyses were performed using SPSS version 24.0 for Windows (IBM Corp., Armonk, NY, USA) and MedCalc version 18.5 (MedCalc Software bvba, Ostend, Belgium).

\section{Ethics statement}

This study was approved by the IRB of Pusan National University Hospital (H-1707-011-061). The requirement for informed consent from the patients was waived due to the observational and retrospective nature of this study.

\section{Results}

During the study period, a total of 18,413 patients were admitted to the six ICUs. Of these, 302 patients (1.6\%) were eligible for the study. The data of these 302 patients were retrieved and included in the analyses. The median age of the cohort was 63 years (range, 18-89 years), and 186 of the cohort (61.6\%) were males. Of the patients, $152(50.3 \%)$ were from surgical departments. In descending order, the three main indications for PMV were neurological disease, pulmonary disease, and multiple traumas. The ICU and hospital mortality rates were $31.5 \%$ and $33.1 \%$, respectively. The demographic and clinical characteristics and the clinical and mortality outcomes of the study cohort are summarized in Table 1.

The median total medical costs per patient during ICU and total hospital stay were 35,105 USD (range, 9,913-204,829 USD) and 41,110 USD (range, 13,605-204,829 USD), respectively. A positive correlation was found between medical costs during ICU stay and LOS in the ICU; and medical costs during total hospital stay and hospital LOS $(\gamma=0.590, p<0.001$ and $\gamma=0.616, p<0.001$, respectively). Medical costs per patient during ICU and hospital stay were negatively correlated with age $(\gamma=-0.177, p=0.002$ and $\gamma=-0.171, p=0.003$, respectively $)$.

Table 2 shows the associations between the ProVent components and medical costs and outcomes during ICU and total hospital stay. Patients aged $<50$ years had higher medical costs and longer LOS (ICU and total hospital) than those in other age groups. Patients with thrombocytopenia on day 21 of PMV had a shorter LOS and higher medical costs during
ICU stay than those without thrombocytopenia on day 21 of PMV. Patients requiring vasopressors on day 21 of PMV had a shorter LOS than patients who did not require vasopressors on day 21 of PMV; however, no significant intergroup differences in medical costs during ICU and total hospital stay were found. Patients requiring hemodialysis on day 21 of MV had higher medical costs (ICU and total hospital stay) than those who did not require hemodialysis on day 21 of PMV.

As the number of these three components (age $<50$ years, thrombocytopenia, and hemodialysis on day 21 of MV) increased, a positive correlation with medical costs was found for both ICU and total hospital stay $(\gamma=0.367, p<0.001$ and $\gamma=0.284, p<0.001$, respectively). A total of 156 patients $(51.7 \%)$ had one of these three ProVent components. Of them, patients

Table 1. Baseline characteristics and outcomes

\begin{tabular}{|lc|}
\hline \multicolumn{1}{|c}{ Variable } & Value (n=302) \\
\hline Age, yr & $63(18-89)$ \\
Male sex & $186(61.6)$ \\
Body mass index, kg/m² & $22.5(14.2-58.6)$ \\
Charlson comorbidity index & $2(0-9)$ \\
Major diagnoses leading to MV & \\
Neurological & $127(42.1)$ \\
Pulmonary, including pneumonia & $69(22.8)$ \\
Multiple traumas & $41(13.6)$ \\
\hline Infections other than pneumonia & $20(6.6)$ \\
Septic shock secondary to infection & $13(4.3)$ \\
Drug intoxication & $12(4.0)$ \\
Cardiogenic pulmonary edema & $11(3.6)$ \\
\hline Postoperative state & $8(2.6)$ \\
Severity of illness on day of ICU admission & \\
\hline APACHE II score & $18(3-41)$ \\
SOFA score & $9(1-16)$ \\
MV LOS, day & $29(21-199)$ \\
ICU LOS, day* & $40(15-201)$ \\
Hospital LOS, day & $50(21-387)$ \\
Tracheostomy during ICU stay & $229(75.8)$ \\
ICU mortality & $95(31.5)$ \\
Hospital mortality & $100(33.1)$ \\
\hline
\end{tabular}

Values presented as average (range) for continuous variables and number (\%) for categorical variables.

*Two patients were maintained receiving MV in a general ward after ICU discharge (ICU LOS of them were 15 and 18 days, respectively).

MV: mechanical ventilation; ICU: intensive care unit; APACHE II: Acute Physiology and Chronic Health Evaluation II; SOFA: Sequential Organ Failure Assessment; LOS: length of stay. 
Table 2. Relationship between components of the ProVent model and total medical costs per person and outcomes during ICU and total hospital stay

\begin{tabular}{|c|c|c|c|c|}
\hline \multirow{2}{*}{ Categorical variable } & \multicolumn{2}{|c|}{ ICU stay } & \multicolumn{2}{|c|}{ Total hospital stay (ICU and general ward) } \\
\hline & Costs (USD) & Length of stay (day) & Cost (USD) & Length of stay (day) \\
\hline \multicolumn{5}{|l|}{ Age, yr } \\
\hline$<50(\mathrm{n}=66)$ & $42,731(13,605-204,829)^{*}$ & $49(21-394)^{*}$ & $48,000(13,605-204,829)^{*}$ & $57(21-387)^{*}$ \\
\hline $50-64(n=97)$ & $35,753(15,337-153,172)^{\dagger}$ & $41(21-155)^{\dagger}$ & $44,775(18,615-174,779)$ & $57(24-384)$ \\
\hline$\geq 65(\mathrm{n}=139)$ & $32,182(9,913-95,888)^{\dagger}$ & $37(15-139)^{\dagger}$ & $37,621(15,496-117,001)^{\dagger}$ & $41(22-227)^{\dagger}$ \\
\hline \multicolumn{5}{|l|}{ Thrombocytopenia } \\
\hline Yes $(n=95)$ & $36,237(9,913-204,829)^{*}$ & $35(18-99)^{\dagger}$ & $40,114(18,985-204,829)$ & $38(22-384)^{\dagger}$ \\
\hline No $(n=207)$ & $34,783(11,111-135,318)^{\dagger}$ & $42(15-394)^{*}$ & $41,286(13,605-135,318)$ & $55(22-387)^{*}$ \\
\hline \multicolumn{5}{|l|}{ Required vasopressors } \\
\hline Yes $(n=83)$ & $32,663(9,913-135,318)$ & $31(18-201)^{\dagger}$ & $37,732(13,605-135,318)$ & $33(21-220)^{\dagger}$ \\
\hline No $(\mathrm{n}=219)$ & $36,156(11,111-204,829)$ & $42(15-394)^{*}$ & $43,191(15,496-204,829)$ & $56(22-387)^{*}$ \\
\hline \multicolumn{5}{|l|}{ Required hemodialysis } \\
\hline Yes $(\mathrm{n}=31)$ & $57,864(19,258-204,829)^{*}$ & $36(23-201)$ & $62,460(19,258-204,829)^{*}$ & $37(24-384)^{\dagger}$ \\
\hline No $(n=271)$ & $33,509(9,913-153,172)^{\dagger}$ & $40(15-394)$ & $39,669(13,605-174,779)^{\dagger}$ & $51(21-387) *$ \\
\hline
\end{tabular}

Values are presented as median (range).

* Values recorded as superscript ${ }^{*}$ have significantly higher medical costs compared to other vaules(s) recorded as superscript ${ }^{\dagger}(\mathrm{p}<0.05)$.

ICU: intensive care unit; USD: U.S. dollars.

Table 3. Relationship between total medical costs per patient and three components (age $<\mathbf{5 0}$, hemodialysis, thrombocytopenia) of the ProVent model

\begin{tabular}{|lcc|}
\hline \multicolumn{1}{|c}{ Component } & ICU stay (USD) & Total hospital stay (USD) \\
\hline None $(\mathrm{n}=146)$ & $32,059(11,111-95,888)$ & $38,444(15,496-109,976)$ \\
\hline One component $(\mathrm{n}=122)$ & $36,284(9,913-153,172)$ & $43,450(13,605-174,779)$ \\
\hline Thrombocytopenia $(\mathrm{n}=62)$ & $32,616(9,913-153,172)^{\dagger}$ & $37,199(18,985-174,779)^{\dagger}$ \\
\hline Age $<50$ yr $(\mathrm{n}=54)$ & $43,543(13,605-122,303)^{\dagger}$ & $48,495(13,605-122,303)^{\dagger}$ \\
\hline Hemodialysis $(\mathrm{n}=6)$ & $55,405(38,995-68,364)^{*}$ & $55,405(38,995-68,364)^{*}$ \\
\hline Two components $(\mathrm{n}=32)$ & $49,814(19,258-135,318)$ & $52,870(19,258-135.318)$ \\
\hline $\begin{array}{l}\text { Age }<50 \text { yr and hemodialysis or thrombocytopenia and } \\
\text { hemodialysis }(\mathrm{n}=23)\end{array}$ & $54,703(19,258-135,318)^{*}$ & $62,460(19,258-135,318)^{*}$ \\
\hline Age $<50$ yr and thrombocytopenia $(\mathrm{n}=9)$ & $37,271(21,846-124,785)^{\dagger}$ & $35,061(21,846-124,785)^{\dagger}$ \\
\hline All three components $(\mathrm{n}=2)$ & $166,317(127,806-204,829)$ & $166,317(127,806-204,829)$ \\
\hline
\end{tabular}

Values are presented as median (range).

* ${ }^{\dagger}$ Values recorded as superscript ${ }^{*}$ have significantly higher medical costs compared to other vaules(s) recorded as superscript ${ }^{\dagger}(\mathrm{p}<0.05)$.

ICU: intensive care unit; USD: U.S. dollars.

requiring hemodialysis had significantly higher medical costs during ICU and total hospital stay than patients with the other components (Table 3 ).

We evaluated the predictive power of the ProVent components for ICU and hospital mortality. Multivariate logistic analyses indicated that three components (requirement for vasopressors, requirement for hemodialysis, and thrombocytopenia [platelet count $<150 \times 10^{9} / \mathrm{L}$ ]) were significantly associated with ICU and hospital mortality (Table 4). According to the $\beta$ coefficient values, the following ProVent model predicting ICU mortality was proposed: requirement for vasopressors ( +2 points), requirement for hemodialysis $(+2$ points), and thrombocytopenia ( +1 point). In addition, the following ProVent model for predicting hospital mortality was proposed: requirement for vasopressors $(+1$ point $)$, requirement for hemodialysis ( +2 points), and thrombocytopenia $(+1$ 
Table 4. Multivariate logistic regression analyses for association between each component of the ProVent model and ICU and hospital mortality

\begin{tabular}{|c|c|c|c|c|c|c|}
\hline \multirow[b]{2}{*}{ Variable } & \multicolumn{3}{|c|}{ ICU mortality } & \multicolumn{3}{|c|}{ Hospital mortality } \\
\hline & $\begin{array}{l}\text { Adjusted OR } \\
(95 \% \mathrm{CI})\end{array}$ & $\beta$ value & p-value & $\begin{array}{l}\text { Adjusted OR } \\
(95 \% \mathrm{CI})\end{array}$ & $\beta$ value & p-value \\
\hline Age $\geq 65 \mathrm{yr}$ & $1.347(0.695-2.609)$ & 0.298 & 0.378 & $1.569(0.822-2.995)$ & 0.451 & 0.172 \\
\hline Age 50-64 yr & $1.057(0.495-2.257)$ & 0.055 & 0.886 & $1.150(0.554-2.388)$ & 0.139 & 0.708 \\
\hline Vasopressors & $4.505(2.476-8.195)$ & 1.505 & $<0.001$ & $3.882(2.157-6.988)$ & 1.356 & $<0.001$ \\
\hline Hemodialysis & $5.429(2.204-14.562)$ & 1.692 & 0.001 & $5.036(1.894-13.393)$ & 1.617 & 0.001 \\
\hline Platelet count $<150 \times 10^{9} / \mathrm{L}$ & $3.755(2.057-6.855)$ & 1.323 & $<0.001$ & $3.495(1.940-6.299)$ & 1.251 & $<0.001$ \\
\hline
\end{tabular}

The Hosmer-Lemeshow goodness-of-fitness statics were $4.578(d f=5, \mathrm{p}=0.470)$ for ICU mortality and $6.928(d f=6$, $\mathrm{p}=0.328)$ for hospital mortality, respectively.

ICU: intensive care unit; OR: odds ratio, CI: confidence interval.

point). The AUCs for the ProVent models for predicting ICU and hospital mortality were 0.787 (95\% confidence interval [CI], 0.729-0.845; $\mathrm{p}<0.001$ ) and 0.769 (95\% CI, 0.709-0.828; $\mathrm{p}<0.001$ ), respectively.

\section{Discussion}

To the best of our knowledge, this is the first comprehensive investigation of the association between medical costs and clinical variables in Korean patients requiring PMV. In light of the financial pressures associated with health-care costs in these patients ${ }^{5}$, we assessed the ability of ProVent models to determine the optimal interventions, optimize resource utilization, and plan for changes to future critical care.

Our results showed that PMV patients require high resource investment during ICU admission, and that three components of the ProVent model (age $<50$ years, and thrombocytopenia and hemodialysis on day 21 of MV) were associated with high medical costs. In addition, three ProVent components (requirement for vasopressors, requirement for hemodialysis, and thrombocytopenia on day 21 of MV) were associated with higher ICU and hospital mortality rates based on multivariate regression analyses. Based on the performance of predicting mortality in previous studies ${ }^{6-9}$, our findings suggest that the ProVent model is predictive of high medical costs and mortality in this population, and as such, may enable prediction of the prognosis and medical costs during ICU and hospital stay ${ }^{7-9}$.

In our enrolled patients, younger patients ( $<50$ years) had higher medical costs than older patients ( $\geq 50$ years), but their ICU ( $25.8 \%$ vs. $33.1 \%$, $\mathrm{p}=0.296)$ and hospital (27.3\% vs. $34.7 \%, \mathrm{p}=0.301$ ) mortality rates were not significantly different. However, patients $<50$ years old had a lower APACHE II score at ICU admission (median, 16 [range 3-36] vs. 18 [6-41]; $\mathrm{p}=0.006$ ). Our findings imply that younger patients tended to use more of various medical resources during their ICU stay.
In addition, among the components of the ProVent model, hemodialysis on day 21 was associated with the highest medical costs. Thus, to predict high medical costs in patients with PMV, attending physicians should evaluate whether patients will require hemodialysis on day 21.

In addition to components of the ProVent model, we hypothesized other clinical and demographic variables could be associated with high medical costs, because staffing levels in the ICUs of university and teaching hospitals in Korea are lower, and critical care delivery systems are underdeveloped compared to Western countries ${ }^{15-17}$. Although the underlying reason is unclear, medical costs per patient during ICU stay were higher in patients who resided outside of Busan (median, 40,842 USD vs. 34,186 USD; $p=0.003$ ). In addition, patients whose main indication for PMV was multiple traumas had higher medical costs per patient than those with the other two main indications for PMV (neurological and pulmonary) (median, 52,320 USD vs. 35,753 USD vs. 52,182 USD; $\mathrm{p}=0.002$ ). However, there was no association between medical costs and other clinical parameters, such as comorbidities (as measured using the Charlson Comorbidity Index) and severity of illness on ICU admission (as measured using the APACHE II and SOFA scores).

Although we could not evaluate in our study due to its retrospective design, medical costs may be influenced by the degree of self-ventilator use at home or at other hospitals at post intensive care settings in patients with PMV. Also, their medical costs may be associated with distinct Korean cultural factors, such as family support, the concept of death, and social customs, as these can impact physician-family discussions regarding life support ${ }^{18}$. Therefore, large-scale, multicenter investigations are needed to evaluate associations between clinical variables and high medical costs in the Korean PMV population.

This study had several limitations. First, no estimates could be made of the medical costs of patients who were not covered by the Korean National Health Insurance coverage 
because of the retrospective nature of the study. Second, we hypothesized that homogeneous subgroup analyses (e.g., underlying diseases, main reasons for ventilator care, and main department at ICU admission) with the ProVent model would be more useful for estimating medical costs, because our study was troubled by heterogeneity resulting from pooling of data from six different ICUs and inter-department health care support systems particularly in surgical, medical, and trauma. We also hypothesized that medical costs could be quite different depending on the level of end-of-life care in these groups of total enrolled patients. However, we did not obtain significant findings, possibly due to the small sample size. Third, the retrospective design of the study may have resulted in selection bias. Fourth, the data were obtained at a single center and the sample size was small, so the results may not be representative of the wider PMV population in Korea.

In conclusion, patients with PMV had high medical costs during ICU and total hospital stay. Three components of the ProVent model (age < 50 years, thrombocytopenia, and hemodialysis on day 21 of MV) were associated with high medical costs during ICU stay. The highest costs were found in patients requiring hemodialysis on day 21 of MV. To facilitate effective resource utilization, further studies to identify predictors of high medical costs in patients requiring PMV are warranted.

\section{Authors' Contributions}

Conceptualization: Lee K. Methodology: All authors. Formal analysis: Roh J, Shin MJ. Data curation: Roh J, Jeong ES. Software: Roh J, Shin MJ. Validation: Lee K, Roh J, Shin MJ. Investigation: Lee K, Roh J, Shin MJ, Jeong ES. Writing - original draft preparation: Lee K, Roh J. Writing - review and editing: Lee K. Approval of final manuscript: all authors.

\section{Conflicts of Interest}

No potential conflict of interest relevant to this article was reported.

\section{Acknowledgments}

This work was supported by the National Research Foundation of Korea (NRF) grant funded by the Korean government (Ministry of Science and ICT) (2016R1C1B1008529).

\section{References}

1. Nelson JE, Cox CE, Hope AA, Carson SS. Chronic critical illness. Am J Respir Crit Care Med 2010;182:446-54.
2. MacIntyre NR, Epstein SK, Carson S, Scheinhorn D, Christopher K, Muldoon S, et al. Management of patients requiring prolonged mechanical ventilation: report of a NAMDRC consensus conference. Chest 2005;128:3937-54.

3. Carson SS. Outcomes of prolonged mechanical ventilation. Curr Opin Crit Care 2006;12:405-11.

4. Donahoe MP. Current venues of care and related costs for the chronically critically ill. Respir Care 2012;57:867-86.

5. Cox CE, Carson SS, Govert JA, Chelluri L, Sanders GD. An economic evaluation of prolonged mechanical ventilation. Crit Care Med 2007;35:1918-27.

6. Carson SS, Garrett J, Hanson LC, Lanier J, Govert J, Brake MC, et al. A prognostic model for one-year mortality in patients requiring prolonged mechanical ventilation. Crit Care Med 2008;36:2061-9.

7. Carson SS, Kahn JM, Hough CL, Seeley EJ, White DB, Douglas IS, et al. A multicenter mortality prediction model for patients receiving prolonged mechanical ventilation. Crit Care Med 2012;40:1171-6.

8. Leroy G, Devos P, Lambiotte F, Thevenin D, Leroy O. Oneyear mortality in patients requiring prolonged mechanical ventilation: multicenter evaluation of the ProVent score. Crit Care 2014;18:R155.

9. Mok JH, Kim YH, Jeong ES, Eom JS, Kim MH, Kim KU, et al. Clinical application of the ProVent score in Korean patients requiring prolonged mechanical ventilation: a 10-year experience in a university-affiliated tertiary hospital. J Crit Care 2016;33:158-62.

10. Acute Respiratory Distress Syndrome Network, Brower RG, Matthay MA, Morris A, Schoenfeld D, Thompson BT, et al. Ventilation with lower tidal volumes as compared with traditional tidal volumes for acute lung injury and the acute respiratory distress syndrome. N Engl J Med 2000;342:1301-8.

11. Charlson ME, Pompei P, Ales KL, MacKenzie CR. A new method of classifying prognostic comorbidity in longitudinal studies: development and validation. J Chronic Dis 1987;40:373-83.

12. Knaus WA, Draper EA, Wagner DP, Zimmerman JE. APACHE II: a severity of disease classification system. Crit Care Med 1985;13:818-29.

13. Vincent JL, Moreno R, Takala J, Willatts S, De Mendonca A, Bruining H, et al. The SOFA (Sepsis-related Organ Failure Assessment) score to describe organ dysfunction/failure. On behalf of the Working Group on Sepsis-Related Problems of the European Society of Intensive Care Medicine. Intensive Care Med 1996;22:707-10.

14. Kim WY, Jo EJ, Eom JS, Mok J, Kim MH, Kim KU, et al. Validation of the Prognosis for Prolonged Ventilation (ProVent) score in patients receiving 14 days of mechanical ventilation. J Crit Care 2018;44:249-54.

15. Kim JH, Hong SK, Kim KC, Lee MG, Lee KM, Jung SS, et al. Influence of full-time intensivist and the nurse-to-patient ratio on the implementation of severe sepsis bundles in Korean 
intensive care units. J Crit Care 2012;27:414.e11-21.

16. Kwak SH, Jeong CW, Lee SH, Lee HJ, Koh Y. Current status of intensive care units registered as critical care subspecialty training hospitals in Korea. J Korean Med Sci 2014;29:431-7.

17. Lim CM, Kwak SH, Suh GY, Koh Y. Critical care in Korea: pres- ent and future. J Korean Med Sci 2015;30:1540-4.

18. Phua J, Joynt GM, Nishimura M, Deng Y, Myatra SN, Chan $\mathrm{YH}$, et al. Withholding and withdrawal of life-sustaining treatments in intensive care units in Asia. JAMA Intern Med 2015; 175:363-71. 